\title{
Dramatic Decrease in Ocular Deviation 1 Day before Surgery in Patients with Intermittent Exotropia
}

\author{
Jinam Lim, Won Jae Kim \\ Department of Ophthalmology, Yeungnam University College of Medicine, Daegu, Korea
}

Purpose: To evaluate the clinical characteristics of patients with intermittent exotropia who exhibited a dramatic decrease in ocular deviation 1 day before surgery.

Methods: This study retrospectively enrolled patients with intermittent exotropia who underwent surgery between December 2013 and December 2019. Those who exhibited a decrease in ocular alignment $\geq 10$ prism diopters (PD) at the last examination ( 1 day before surgery) compared with the largest previous angle of deviation were included. A monocular occlusion test was performed to re-confirm the largest angle of ocular deviation.

Results: Among 547 patients with intermittent exotropia, 10 (six females; mean age, 8.6 years) exhibited a dramatic decrease in ocular deviation before impending surgery. The mean largest angle of deviation was 31.0 PD (range, 20 to 50 PD) at distance and 34.5 PD (range, 20 to 55 PD) at near. The mean control scores using the LACTOSE (Look and Cover then Ten Seconds of Observation Scale for Exotropia) scoring system were 2.5 at distance, 1.8 at near, and 4.3 when combined. Mean ocular deviation prior to the impending surgery decreased to 7.4 PD (range, 0 to 10 PD) at distance and 6.2 PD (range, -10 to 10 PD) at near. The largest angle of ocular deviation was re-confirmed using the monocular occlusion test in all patients. All patients underwent surgery as planned, and none exhibited postoperative overcorrection.

Conclusions: A relatively small number of patients with intermittent exotropia exhibited a dramatic decrease in ocular alignment 1 day before surgery but demonstrated a relatively better level of control. The monocular occlusion test was helpful in re-confirming the largest angle of ocular deviation.

Key Words: Anxiety, Exotropia, Surgery

The angle of ocular deviation can be variable according to level of control as measured during follow-up examinations in patients with intermittent exotropia [1,2]. Determining the largest angle of ocular deviation and designat-

Received: March 4, 2020 Final revision: May 4, 2020

Accepted: May 14, 2020

Corresponding Author: Won Jae Kim, MD. Department of Ophthalmology, Yeungnam University College of Medicine, 170 Hyeonchung-ro, Nam-gu, Daegu 42415, Korea. Tel: 82-53-620-3444, Fax: 82-53-626-5936, E-mail: eyekwj@ynu.ac.kr ing it as the target for surgery is necessary to achieve favorable surgical outcomes in patients with intermittent exotropia $[1,3,4]$. Therefore, alternative methods, such as the monocular occlusion test, repeated measurements, and outdoor measurement, have been used to reveal the largest angle of ocular deviation [1].

The level of control in patients with intermittent exotropia can be affected not only by extrinsic factors, but also by intrinsic factors such as general health, alertness, attention span, and level of anxiety [5]. Pediatric patients sched- 
uled to undergo anesthesia and surgery usually report significant anxiety [6,7]. Although rare, a dramatic decrease in ocular deviation caused by anxiety associated with impending surgery can also occur in patients with intermittent exotropia [5]. It is recommended to perform the surgery as planned; however, the risks for postoperative overcorrection should be considered by even the most experienced surgeons [5]. To our knowledge, no previous studies have investigated the characteristics of patients who exhibit a dramatic decrease in ocular deviation preoperatively. Accordingly, we examined patients with intermittent exotropia who exhibited a dramatic decrease in ocular deviation 1 day before their scheduled surgery.

\section{Materials and Methods}

The present study adhered to the tenets of the Declaration of Helsinki and was approved by the institutional review board of Yeungnam University Hospital (2019-12045). Given the retrospective nature of the study and the use of anonymized patient data, requirements for informed consent were waived by the board. This study retrospectively enrolled patients with intermittent exotropia who underwent surgical treatment between December 2013 and December 2019. Patients with intermittent exotropia who exhibited a decrease in ocular alignment $\geq 10$ prism diopters (PD) at both distant and near fixation compared with the largest angle of deviation at the last examination (1 day before surgery) were included in the study. Individuals with any other types of strabismus, such as oblique muscle dysfunction, dissociated vertical deviation, an A-V pattern, or nystagmus, were excluded. Individuals who had undergone previous intraocular surgery and those with any neurological impairment or severe unilateral amblyopia were also excluded.

\section{Preoperative evaluation}

At the initial visit, all patients underwent a full ophthalmologic examination, including testing of visual acuity and ocular alignment status, assessment of level of control, slitlamp biomicroscopy, refraction, fundus examination, and stereoacuity testing. Best-corrected visual acuity was measured, when possible. If patients were considered too young for optotype testing at the initial visit, visual acuity was evaluated based on fixation preference. The angle of deviation was measured both pre- and postoperatively using an alternate prism cover test at 6-m and 33-cm fixation in cooperative patients. An ocular patch was provided to the patients at the first visit, and they were instructed to occlude the non-dominant eye for 1 hour at the next visit to measure the largest angle of deviation. The level of control was assessed using the Look and Cover then Ten Seconds of Observation Scale for Exotropia (LACTOSE) control scoring system [8]. This control system was developed by incorporating scales for both distance and near evaluations (5-point scales, scored from 0 to 4 each), yielding a total score ranging from 0 to 8 . A higher score indicates a poorer level of control. Controllability was defined as the patient's subjective awareness of exotropia or diplopia associated with presence of exotropia and ability to instinctively correct ocular deviation. More than two preoperative examinations were performed on each patient before surgery. Stereoacuity measurements were carried out using the Lang I (Lang-Stereotest AG, Küsnacht, Switzerland) and Stereo Fly Stereotest (Stereo Optical, Chicago, IL, USA) when the patient was able to cooperate and complete the test.

\section{Final examination of ocular alignment before surgery}

Patients were admitted to the hospital 1 day before surgery according to the authors' preoperative protocol. The final examination of ocular alignment was performed on

Table 1. Surgical amount of BLR, and unilateral lateral rectus recession and medial $R \& R$ for patients with intermittent exotropia

\begin{tabular}{lcccc}
\hline \multirow{2}{*}{ Prism diopters } & \multicolumn{2}{c}{ BLR } & & \multicolumn{2}{c}{ R\&R } \\
\cline { 2 - 3 } \cline { 5 - 5 } & $\begin{array}{c}\text { Bilateral } \\
\text { recession } \\
\text { amount of LR }\end{array}$ & & $\begin{array}{c}\text { Recession } \\
\text { amounts of } \\
\text { LR }\end{array}$ & $\begin{array}{c}\text { Resection } \\
\text { amounts of } \\
\text { MR }\end{array}$ \\
\hline 20 & 5 & - & - \\
25 & 6 & & 4 & 3 \\
30 & - & & 4 & 4 \\
35 & - & 5 & 4 \\
40 & - & & 5 & 5 \\
45 & - & & 7 & 5 \\
50 & - & 8 & 5 \\
\hline
\end{tabular}

$\mathrm{BLR}=$ bilateral lateral rectus recession; $\mathrm{R} \& \mathrm{R}=$ rectus resection; $\mathrm{LR}=$ lateral rectus muscle; $\mathrm{MR}=$ medial rectus muscle . 
the evening before surgery (usually between 6:00 and 7:00 p.m.). If any patients exhibited a dramatic decrease in ocular deviation compared with the largest angle obtained during previous measurements, a 30-minute monocular occlusion test was conducted to confirm the largest ocular deviation. Additionally, a 1-hour monocular occlusion test was performed if the 30-minute monocular occlusion test failed to reveal the largest ocular deviation. All measurements of ocular deviation were performed by the same clinician (WJK). The surgery was carried out using the surgical protocol developed at the authors' clinic (Table 1). The angle of deviation measured during the first follow-up visit within 1 week of surgery was defined as the immediate postoperative deviation. The patients were followed-up at $1,3,6$, and 12 months after the surgery and then every 6 months thereafter.

\section{Statistical analysis}

Continuous data are expressed as mean with standard deviation, and categorical data are shown as count and percentage. Patient data, including sex, age at surgery, largest angle of ocular deviation, level of control, controllability, decrease in ocular deviation at the final examina- tion, and surgical outcomes, were collected. Data were analyzed using the IBM SPSS Statistics ver. 20.0 (IBM Corp., Armonk, NY, USA).

\section{Results}

\section{Basic characteristics of patients with intermittent exo- tropia}

This study retrospectively reviewed 547 patients with intermittent exotropia who underwent surgical treatment during the study period. Among these participants, 10 (6 females) exhibited a decrease in ocular alignment $\geq 10$ PD compared with the largest angle of deviation obtained at the last examination $(1.8 \%, 10 / 547)$. The basic characteristics of the included patients are shown in Table 2. The mean age of the included patients was $8.5 \pm 2.1$ years (range, 5 to 13 years). Eight patients had basic type of intermittent exotropia. Two patients (patients 6 and 8 ) demonstrated pseudo-divergence excess type of intermittent exotropia. The level of control achieved as assessed using the LACTOSE scoring system was measured for eight included patients. The mean control scores were 2.5

Table 2. Basic characteristics of patients who exhibited dramatic decrease in ocular alignment 1 day before surgery

\begin{tabular}{|c|c|c|c|c|c|c|c|c|}
\hline \multirow{2}{*}{ Patient } & \multirow{2}{*}{$\begin{array}{l}\text { Sex / age } \\
(y r)\end{array}$} & \multicolumn{2}{|c|}{$\begin{array}{l}\text { Angle of deviation } \\
\text { at the initial visit (PD) }\end{array}$} & \multirow{2}{*}{ Controllability } & \multicolumn{2}{|c|}{$\begin{array}{l}\text { Control score } \\
\text { (LACTOSE) }\end{array}$} & \multicolumn{2}{|c|}{ Stereoacuity } \\
\hline & & Distant & Near & & Distant & Near & Lang I & $\begin{array}{c}\text { Stereo Fly Stereotest } \\
\text { (arcsec) }\end{array}$ \\
\hline 1 & $\mathrm{~F} / 9$ & 20 & 20 & Yes & - & - & Pass & 80 \\
\hline 2 & $\mathrm{~F} / 9$ & 30 & 20 & Yes & - & - & Pass & 60 \\
\hline 3 & $\mathrm{M} / 7$ & 30 & 30 & No & 4 & 2 & Pass & 60 \\
\hline 4 & $\mathrm{M} / 8$ & 30 & 35 & No & 4 & 1 & Pass & 40 \\
\hline 5 & $\mathrm{M} / 9$ & 30 & 30 & No & 1 & 1 & Pass & 100 \\
\hline 6 & $\mathrm{~F} / 13$ & 40 & 25 & Yes & 1 & 1 & Pass & 40 \\
\hline 7 & $\mathrm{~F} / 8$ & 30 & 30 & Yes & 1 & 0 & Pass & 80 \\
\hline 8 & $\mathrm{~F} / 7$ & 30 & 10 & Yes & 3 & 3 & Pass & 40 \\
\hline 9 & $\mathrm{~F} / 9$ & 18 & 18 & Yes & 2 & 2 & Pass & 40 \\
\hline 10 & $\mathrm{M} / 5$ & 25 & 25 & No & 4 & 4 & Pass & 400 \\
\hline
\end{tabular}

The level of control was measured using the LACTOSE control scoring system. This control system was constructed by incorporating scales for both distant and near evaluations (5-point scales: responses ranging from 0 to 4 on each), yielding a total score that ranged from 0 to 8. A higher score indicated a poorer level of control. Controllability was defined as the patient's subjective awareness of exotropia or diplopia associated with exotropia and the ability to instinctively correct the ocular deviation.

$\mathrm{PD}=$ prism diopters; LACTOSE $=$ Look and Cover then Ten Seconds of Observation Scale for Exotropia. 
Table 3. Clinical features of patients with intermittent exotropia who exhibited dramatic decrease in ocular deviation 1 day before surgery

\begin{tabular}{|c|c|c|c|c|c|c|c|}
\hline \multirow[t]{2}{*}{ Patient } & \multicolumn{2}{|c|}{$\begin{array}{l}\text { Largest angle of deviation during } \\
\text { follow-up (PD) }\end{array}$} & \multicolumn{2}{|c|}{$\begin{array}{l}\text { Ocular deviation } \\
\text { at the final examination before } \\
\text { surgery (PD) }\end{array}$} & \multicolumn{2}{|c|}{$\begin{array}{l}\text { Ocular deviation at the } \\
\text { final visit after surgery }\end{array}$} & \multirow[t]{2}{*}{$\begin{array}{l}\text { Follow-up } \\
\text { length (mon) }\end{array}$} \\
\hline & Distant & Near & Distant & Near & Distant & Near & \\
\hline 1 & 20 & 20 & Ortho & Ortho & 4 & 4 & 39 \\
\hline 2 & 30 & 30 & 8 & 16 & 4 & 4 & 18 \\
\hline 3 & 30 & 35 & 12 & 10 & 20 & 20 & 16 \\
\hline 4 & 30 & 40 & 10 & 10 & 6 & 4 & 18 \\
\hline 5 & 30 & 35 & 16 & 16 & 4 & Ortho & 12 \\
\hline 6 & 40 & 40 & 10 & 12 & 12 & 14 & 9 \\
\hline 7 & 35 & 40 & Ortho & Ortho & 4 & 4 & 6 \\
\hline 8 & 50 & 55 & Ortho & -10 & 2 & 2 & 12 \\
\hline 9 & 20 & 25 & 10 & Ortho & 2 & 2 & 9 \\
\hline 10 & 25 & 25 & 8 & 8 & 2 & 6 & 3 \\
\hline
\end{tabular}

The positive numbers represent exodeviation, and the negative numbers represent esodeviation.

$\mathrm{PD}=$ prism diopters; Ortho $=$ orthotropia.

\pm 1.4 at distant fixation, $1.8 \pm 1.3$ at near fixation, and $4.3 \pm$ 2.4 when combined. Six patients $(60.0 \%, 6 / 10)$ exhibited controllability of exotropia. The mean spherical equivalent refractive errors of the included patients were $-0.59 \pm 1.12$ diopters ( $D$; range, -2.25 to +1.00 ) in the right eye and -0.65 $\pm 1.02 \mathrm{PD}$ (range, -2.00 to +1.00 ) in the left eye.

\section{Decrease in ocular deviation 1 day before surgery and the effect of the monocular occlusion test}

The largest angles of ocular deviation, initial ocular deviation at the final examination, and ocular deviation 1 day before surgery are summarized in Table 3. The mean largest angle of deviation was $31.0 \pm 9.1 \mathrm{PD}$ (range, 20 to 50 PD) at distant fixation and $34.5 \pm 10.1 \mathrm{PD}$ (range, 20 to 55 PD) at near fixation. The mean angle of ocular deviation decreased to $7.4 \pm 5.6 \mathrm{PD}$ (range, 0 to $16 \mathrm{PD}$ ) at distant fixation and 6.2 $\pm 8.4 \mathrm{PD}$ (range, -10 to $16 \mathrm{PD}$ ) at near fixation 1 day before surgery. The largest decrease in ocular deviation was $50 \mathrm{PD}$ at distance and $65 \mathrm{PD}$ at near.

The 30-minute monocular occlusion test was performed in all patients to re-check the largest angle of deviation, with nine patients exhibiting an increase in ocular deviation, the same number compared with the previous largest angle of ocular deviation. One patient (patient 8) who exhibited the largest decrease in ocular deviation required an additional 1-hour monocular occlusion test to confirm the largest ocular deviation. All patients underwent surgical treatment as planned. During postoperative follow-up examinations (range, 3 to 39 months), no patients exhibited postoperative overcorrection of exotropia.

\section{Discussion}

In the present study, $1.8 \%$ of all included patients with intermittent exotropia exhibited a dramatic decrease in ocular alignment $\geq 10$ PD 1 day before surgery compared with the largest angle of deviation measured previously. These patients exhibited a relatively better level of control. Patients with controllability demonstrated a higher possibility of dramatic decreases in ocular alignment prior to an impending surgery. The monocular occlusion test was helpful in re-confirming the largest angle of ocular deviation. The surgery could therefore continue as planned without concerns about postoperative overcorrection.

Patients with intermittent exotropia may exhibit variable degrees of ocular deviation according to level of control during follow-up. A previous study demonstrated that more than half of patients with intermittent exotropia $(62.8 \%, 216$ / 344) exhibit variable angles of deviation during preoperative measurements [2]. von Noorden and 
Campos [5] described patients with intermittent exotropia who displayed a dramatic decrease in ocular deviation caused by anxiety associated with impending surgery. This anxiety may increase the level of patient control and lead to straight ocular alignment in patients with intermittent exotropia. It is recommended to perform the surgery as planned [5]; however, it sometimes may be difficult for the surgeon to decide on the best surgical strategy due to the possibility of postoperative overcorrection. No previous study has evaluated patients with intermittent exotropia who exhibited dramatic decreases in ocular deviation before impending surgery.

In the present study, a relatively small number of patients with intermittent exotropia exhibited a dramatic decrease in ocular deviation 1 day before surgery. These results are similar to those reported by Seo and Chung [9], who demonstrated that the angle of deviation at distance on the day of surgery was decreased in $1.9 \%(6 / 323)$ and the near angle was decreased in $6.8 \%(22 / 323)$ of patients. Patients who exhibited decreased near deviation were older than those with no change, and $81.8 \%$ of the patients were school-aged. Seo and Chung [9] postulated that older pediatric patients may be frightened by the prospect of surgery. In our study, the mean age of included patients was 8.5 years, which is old enough to be afraid of the prospect of surgery. Wariness of surgery may increase anxiety, which in turn leads to a temporal increase in level of control and a decrease in ocular deviation. One patient who exhibited the largest decrease in ocular deviation postponed the surgery one time due to extreme worry. She was also so anxious that she exhibited esotropia at near fixation at the last examination.

Patients with a relatively better level of control can exhibit dramatic changes in ocular deviation with impending surgery. In our study, the mean control score was lower, reflecting a better level of control compared with a previous study that included pediatric patients with intermittent exotropia [8]. The included patients showed good stereoacuity. Patients with relatively better fusional potential are considered more likely to maintain a period of normal ocular alignment but can easily mask the full amount of ocular deviation, leading to the observed dramatic decrease in ocular deviation [2]. More than one-half of included patients demonstrated controllability-a subjective awareness of exotropia. The level of control in patients with controllability may be more vulnerable to intrinsic factors such as anxiety. All examinations in the present study were performed by the same clinician in the same clinical settings, including fixation distance and accommodative targets. Therefore, the effect of extrinsic factors such as examiner, fixation distance, target size, and room luminance on the level of control should have had little influence on the results [5].

Based on our results, surgery can be performed in these patients as planned. Although patients with intermittent exotropia can exhibit a dramatic decrease in ocular deviation before surgery, spontaneous improvement of ocular deviation is rare in the natural course of intermittent exotropia $[10,11]$. We attempted to re-confirm the largest angle of ocular deviation using an alternate method, and the surgery was performed as planned. The study by Seo and Chung [9] demonstrated that the rate of postoperative overcorrection may be high in patients with decreased near deviation on the day of surgery. However, no patients in our study experienced postoperative overcorrection. We postulate that these differences can be attributed to differences in preferred surgical procedure and target of surgery. Previous studies only included patients who underwent bilateral lateral rectus recession. We performed the bilateral lateral rectus recession procedure or unilateral lateral rectus recession and medial rectus resection based on surgeon preference after re-confirmation of the largest angle of ocular deviation.

To re-confirm ocular alignment, a 30-minute monocular occlusion test was performed in all patients. The monocular occlusion test is used to differentiate the type of exotropia between true and pseudo-divergence excesses by blocking fusional stimuli and disruption of the tonic convergence [1]. Furthermore, this test can determine the largest ocular deviation in patients with intermittent exotropia. The 30-minute monocular occlusion test, which is less time-consuming than the hour-long method, was adequate for revealing the largest angle of ocular deviation, except in one patient. A 1-hour monocular occlusion test was required to re-confirm the largest angle of ocular deviation in this patient. Therefore, the 30-minute monocular occlusion test may be helpful in re-confirming the extent of ocular deviation in patients who exhibit a dramatic decrease in ocular deviation prior to surgery.

In addition to our results, methods to mitigate anxiety due to impending surgery should be considered for patients with intermittent exotropia. Although the level of 
control and amount of ocular deviation can be affected by anxiety associated with impending surgery, alternative methods of calming patients with anxiety have not been extensively studied in our field. Recently, a preoperative preparation program focusing on reducing anxiety before surgery was developed for pediatric patients $[12,13]$. The adaptation of this program and its effect on the angle of ocular deviation may be considered as a future topic of investigation.

The present study had several limitations. First, psychological test(s) to assess the degree of anxiety in our patients were not performed due to the retrospective nature of the study. Psychological tests that reflect an individual's personality may be helpful in understanding the characteristics of patients who exhibit a dramatic decrease in ocular deviation before impending surgery. Second, the influence of parents on the patient anxiety was not considered in this study. Parental anxiety is strongly related to child preoperative anxiety and postoperative pain [14]. It is not uncommon for the parents of pediatric patients with preoperative anxiety to also be very anxious about the surgery. The effect of parental anxiety on pediatric patients with intermittent exotropia may be considered as a future topic of investigation.

\section{Conflict of Interest}

No potential conflict of interest relevant to this article was reported.

\section{References}

1. Kushner BJ. Strabismus. 1st ed. Gewerbestrasse: Springer; 2017. p. 73-95.

2. Kim WJ, Kim MM. Variability of preoperative measurements in intermittent exotropia and its effect on surgical outcome. J AAPOS 2017;21:210-4.

3. Kim WJ, Kim MM. The clinical course of recurrent intermittent exotropia following one or two surgeries over 24 months postoperatively. Eye (Lond) 2014;28:819-24.

4. Kim WJ, Kim MM. The fast exodrift after the first surgical treatment of exotropia and its correlation with surgical outcome of second surgery. BMC Ophthalmol 2018;18:67.

5. von Noorden GK, Campos EC. Binocular vision and ocular motility. 6th ed. St. Louis: Mosby; 2002. p. 356-76.

6. Kain ZN, Mayes LC, O'Connor TZ, Cicchetti DV. Preoperative anxiety in children: predictors and outcomes. Arch Pediatr Adolesc Med 1996;150:1238-45.

7. Waldschmidt B, Gordon N. Anesthesia for pediatric ophthalmologic surgery. J AAPOS 2019;23:127-31.

8. Kim H, Kim DH, Ahn H, Lim HT. Proposing a new scoring system in intermittent exotropia: towards a better assessment of control. Can J Ophthalmol 2017;52:235-9.

9. Seo EJ, Chung SA. Variability of preoperative angle of deviation measured on the day of surgery in intermittent exotropia. J Korean Ophthalmol Soc 2015;56:1591-8.

10. Chia A, Seenyen L, Long QB. A retrospective review of 287 consecutive children in Singapore presenting with intermittent exotropia. J AAPOS 2005;9:257-63.

11. Nusz KJ, Mohney BG, Diehl NN. The course of intermittent exotropia in a population-based cohort. Ophthalmology 2006;113:1154-8.

12. Millett CR, Gooding LF. Comparing active and passive distraction-based music therapy interventions on preoperative anxiety in pediatric patients and their caregivers. $J$ Music Ther 2018;54:460-78.

13. Perry JN, Hooper VD, Masiongale J. Reduction of preoperative anxiety in pediatric surgery patients using age-appropriate teaching interventions. J Perianesth Nurs 2012;27:6981.

14. Fortier MA, Kain ZN. Treating perioperative anxiety and pain in children: a tailored and innovative approach. Paediatr Anaesth 2015;25:27-35. 